\title{
Construcciones críticas sobre las prácticas pedagógicas: reflexiones a partir de la psicología cultural y discursiva*
}

\author{
Critical construction of pedagogical practices: reflections from cultural and discursive \\ psychology \\ Construção crítica das práticas pedagogicas: reflexões sobre a psicologia cultural e \\ discursiva
}

\section{Esperanza Mendoza M., ${ }^{a}$ William Buitrago T., ${ }^{b}$ Luis Felipe González-Gutiérrez ${ }^{c}$}

${ }^{a}$ Fundación Universitaria los Libertadores. Cra. 16 N 63A68. Sede Ricaurte, Ofic 213. Bogotá - Colombia.

Correo electrónico: emendozam@libertadores.edu.co

bCorporación Universitaria Minuto de Dios. Correo electrónico: williambuitragotorres@yahoo.es

cUniversidad Santo Tomás. Correo electrónico: luisgonzalez@usantotomas.edu

\begin{abstract}
RESUMEN
Este artículo presenta una reflexión crítica sobre las prácticas pedagógicas a partir de algunas premisas centradas en la psicología cultural (Potter, 2008; González-Rey, 2009), además de los postulados del construccionismo social (Gergen, 2006, 2011), gracias a las cuales se pueden comprender los procesos educativos como construcciones sociales, mediadas históricamente, que permiten comprender las complejas relaciones que se dan entre estudiantes, docentes, tecnologías involucradas en el proceso educativo y el sistema de redes y comunidades que interactúan en el proceso de enseñanza aprendizaje. La reflexión generada en este texto implica el reconocimiento de la práctica pedagógica como acción mediata, centrada en las relaciones interpersonales y contextualizada en el discurso que permea las instituciones educativas como organismos vivos en permanente transformación.
\end{abstract}

Palabras clave: práctica pedagógica, psicología cultural, construccionismo social, análisis del discurso.

\begin{abstract}
This article presents a critical reflection on pedagogical practices from some premises centered of cultural psychology (Potter, 2008, Gonzalez-Rey, 2009), in addition to the postulates of social constructionism (Gergen, 2006, 2011), thanks to which may include educational processes as social constructs, mediated historically, that allow us to understand the complex relationships that exist between students, teachers, technologies involved in the educational process and the system of networks and communities that interact in the teaching-learning process. The reflection generated in this text, implies the recognition of pedagogical practice as mediate action, focusing on interpersonal relationships, contextualized discourse that permeates educational institutions, as living organisms, in constant transformation.
\end{abstract}

Key words: pedagogical practice, cultural psychology, social construccionism, discourse analysis.

\section{RESUMO}

Este artigo apresenta uma reflexão crítica sobre as práticas pedagogicas a partir de alguns instalações centradas no psicologia cultural (Potter, 2008, Gonzalez-Rey, 2009), também dos princípios do construcionismo social (Gergen, 2006, 2011), através do qual pode incluir processos educativos como construções sociais, mediada historicamente,

* $\quad$ Este artículo hace parte de los resultados del proyecto de investigación: "Impacto en las prácticas pedagógicas del egresado de la especialización pedagogía de la Lúdica para el Desarrollo Cultural”, proyecto que pertenece a la Especialización en Pedagogía de la Lúdica para el Desarrollo Cultural. Facultad de Ciencias de la Educación y financiado por la Fundación Universitaria Los Libertadores. El proyecto se circunscribe a la línea de investigación: Pedagogía Medios y Mediaciones. 
que nos permitem compreender as complexas relações que existem entre alunos, professores, tecnologias envolvidas no processo educativo e do sistema de redes e comunidades que interagem no processo de ensino-aprendizagem. A reflexão gerada neste texto, implica o reconhecimento da prática pedagogica como açao mediada, centralizado nas relações interpessoais e dada na discurso que permeia as instituições de ensino, como estruturas vivas, em constante transformação.

Palavras chave: prática pedagógica, psicología cultural, construccionismo social, análise do discurso.

\title{
1. ALGUNAS CONSIDERACIONES SOBRE PRÁCTICAS PROFESIONALES
}

Gracias al interés que suscitan los procesos de formación, los diferentes elementos sociales y culturales que se ponen en juego hacen de las prácticas pedagógicas un tema de discusión y de análisis para reafirmar la importancia de la formación de los docentes, de la correspondencia que tienen ellos con los diferentes sistemas que se involucran en el proceso educativo y, en consecuencia, el poder transformador que tienen estas prácticas pedagógicas.

Es así que la práctica pedagógica ha sido abordada por diferentes autores (como se verá más adelante) que han destacado, entre otras características, la acción, la práctica, el contexto social involucrado, la relación con la comunidad académica y el espacio flexible de sus acciones, además de elementos fundamentales para la relación con los estudiantes, expresadas en procesos de cooperación, reflexión, diálogo colaborativo, entre otros. De acuerdo a estas características, las prácticas pedagógicas, hoy en día deben ser comprendidas en un mundo cargado de nuevas experiencias relacionales, mediadas no solo en la práctica misma y sus definiciones, sino en sus consecuencias pragmáticas, expresadas en sus niveles comprensivos y descriptivos, además de las exigencias en un mundo mediado también por las tecnologías de la información y la comunicación.

Para iniciar con algunas de estas consideraciones teóricas sobre las prácticas pedagógicas, se destacan los aportes de Latorre (2004), quien hace énfasis en la necesidad de identificar la palabra acción y la palabra práctica, que se expresa de la siguiente forma:

\begin{abstract}
En el contexto de esta investigación (proyecto de investigación: "Formación inicial y prácticas pedagógicas: desafíos pendientes. Aportes para el mejoramiento de la calidad y equidad de la educación chilena (2003-2005)"), la práctica educativa y, más específicamente, la práctica pedagógica serán consideradas desde una doble perspectiva, tanto desde la acción realizada como desde el sentido atribuido a dicha acción por quien la realiza. Esta doble entrada hará posible articular un nivel descriptivo con otro comprensivo (2004: 87).
\end{abstract}

De acuerdo con lo anterior, se ha visto que la acción se entiende en términos de las consecuencias de las actuaciones de las personas en el nivel del análisis, mientras que la práctica, involucra elementos de comprensión que van más allá de dichas acciones descriptivas, lo que puede expresarse en la consideración de dos momentos paradigmáticos; el primero, la acción, centrada en un nivel explicativo de la realidad; y un segundo, el de la práctica, centrada en el nivel interpretativo de la realidad.

Es así como, además de la acción y la práctica - en su definición- es necesario observar las acciones como un conjunto propio de cada individuo, que se manifiesta en las prácticas pedagógicas, tal como lo nombra Latorre (2004): "el objetivar las racionalidades presentes en las prácticas pedagógicas hará posible dar cuenta del mecanismo estructurador $\mathrm{u}$ organizador del conjunto de acciones que realiza un individuo y que se expresa concretamente a través de las acciones que este realiza" (2004: 88). 
Esta tesis que plantea Latorre explica incluir el concepto de racionalidad como la posibilidad de comprender la acción que el docente realiza en su contexto, lo que explica comprensivamente la práctica pedagógica situada y en permanente actualización. Esto brinda la relación complementaria entre las lectura descriptivas sobre la realidad, a la vez que se expresan las características interpretativas que subyacen a las descripciones iniciales.

Por otro lado, De Vicenzi (2009) plantea, en un estudio realizado a docentes universitarios del programa de medicina en una universidad argentina, que el 64\% de la población estudiada centran en la mediación de sus significados la actuación de sus prácticas pedagógicas, debido al sentido que implica el relacionar la experiencia docente con los procesos cognitivos que sustentan la información y su sometimiento a las condiciones del entorno sociocultural. Esto, en palabras del autor, se expresa así: "Este tipo de prácticas docentes rescata la importancia de reconocer, tanto en el docente como en los alumnos, su capacidad para procesar la información a la que somos sometidos en cada instante por la interrelación que se produce con el contexto social" (2009: 98).

Este argumento de la relación complementaria del entorno y la experiencia cognitiva, guarda una relación directa con lo planteado anteriormente por Latorre (2004), pues en la expresión descriptiva de la realidad (la palabra acción) se requiere de un proceso cognitivo que lo sustente y en el nivel comprensivo de la realidad (el sentido de la palabra) requiere de una serie de lectura e interpretaciones, que deben involucrar necesariamente el contexto social para la producción de sentido.

$\mathrm{Y}$ es frente a las relaciones que se establecen con el contexto social, expresado en actividades con sentido y estructura, que se hace conveniente retomar la investigación realizada por Quiroz (2006), en la que reconoce el papel del pensamiento crítico pedagógico para la formación de los estudiantes. Dicho pensamiento, caracterizado por la expresión estructurada de actividades, a partir de contenidos de formación pedagógica, debe ser iniciada en un plano social, para luego derivarse en actividades individuales de aprendizaje y siempre en reflexión crítica con los factores sociales y culturales; esto permite asegurar el sentido activo del estudiante, así como la expresión de nuevos sentidos de interpretación sobre problemas sociales.

En este aspecto otro autores, como López-Vargas y Basto-Torrado (2010), establecen los aportes de una mirada centrada a las prácticas reflexivas y cognitivas para dar cuenta del poder transformador de las prácticas pedagógicas. La colaboración, el sentido de relación y la inserción del concepto de habitus de la teoría del arte, en tanto poder creador y reiteración de los modos de relación dentro del aula, permiten comprender la formación que deben tener los docentes. En palabras de los autores:

El buen profesor de hoy requiere, entonces, una formación que le permita aplicar en su ejercicio docente lo que desde la Sociología de la Educación se ha llamado pedagogías productivas, aquellas que enfatizan en la naturaleza construida del conocimiento, en las múltiples perspectivas sobre la realidad y en una aproximación constructivista y colaborativa del aprendizaje (2010: 287).

Desde la perspectiva que se ofrece aquí, la descripción-comprensión de la realidad, expresada en la práctica pedagógica, implica que el conocimiento tiene una naturaleza social que debe ser armada; por decirlo así, a partir de las experiencias compartidas y colaborativas de los estudiantes, además de la experticia del docente en recrear y ofrecer estas múltiples lecturas sobre el fenómeno de aprendizaje que se está abordando. También debe ser un esfuerzo de las diferentes instituciones educativas, en la promoción de estos 
niveles de confianza y fortalecimiento por el diálogo creativo, expresado en nuevas relaciones. López-Vargas y Basto-Torrado lo resumen en estos términos: "El esfuerzo de las instituciones educativas debe centrarse, entonces, en asegurar un proceso permanente de formación de un pensamiento crítico en el profesorado, que le permita crear las condiciones para su autorregulación mediante el desarrollo de la metacognición como punto de partida" (2010: 288).

Resulta valioso, como se ha visto, la permanente necesidad de interactuar desde procesos cognitivos básicos, además de sus correspondencias creadas con otras partes del sistema educativo, que pasan desde la institución educativa hasta el contexto mismo del que surgen las descripciones e interpretaciones sobre el proceso educativo. En otras palabras, debe traerse la experiencia comunitaria al escenario educativo, además de contar con el sentido auto referencial de las actuaciones del docente y de la institución educativa.

Es así como las prácticas pedagógicas necesariamente invocan el sentido de la comunidad que las alberga, alimenta y sostiene. Estas relaciones interconectadas permiten comprender el impacto de la práctica desde la lógica de la institución, de las comunidades académicas de donde surge la experiencia de los docentes y, en consecuencia, las recompensas sobre la calidad de la educación y el reconocimiento de las acciones concretas de los docentes. Esto se ve reflejado en los argumentos de Francis y Marín (2010) cuando afirman:

\begin{abstract}
...se reconoce que el papel de la comunidad profesional académica tiene un papel fundamental en la construcción de las nociones de academia: cómo se comporta un profesor universitario, cuáles valores son los que fundan su acción y la de sus pares. En este sentido, las prácticas docentes y los saberes pedagógicos que les sirven de base se convierten en contenidos implícitos con un papel reducido, pero permeado por el discurso de la academia (2010: 28).
\end{abstract}

A este respecto la comunidad académica juega un papel de gran importancia por su acción específica, que da sentido a los contenidos, que si bien se ven reducidos, se restablecen con la construcción social que se realiza dentro de la comunidad, que fundamenta y valora la acción de los docentes. En consecuencia, la acción de la práctica es permeada gracias a las enunciaciones discursivas (tanto sociales y culturales) que atraviesan la experiencia de las comunidades, lo cual tiene sentido desde una perspectiva del análisis del discurso educativo, en el que se entiende el conocimiento como uno de los dispositivos en el que las relaciones de poder se mantienen, actualizan y complementan, gracias a la correspondencia colaborativa de significados (Férnandez, 2005; Martínez-Otero, 2010; Prados y Cubero, 2005; Tuñón y Pérez, 2009). Si bien se colocan ejemplos de algunas reflexiones teóricas sobre esta relación discursiva en el plano educativo, el tema es tan vasto que requeriría comentarios para un texto diferente al presentado aquí. De todos modos, en la discusión del texto se enunciarán algunos esbozos que sirvan de abrebocas para la relación indiscutible entre las perspectivas de análisis de discurso, mediadas por la psicología cultural, para la comprensión cualitativa de las prácticas pedagógicas.

Además de la comunidad académica, la percepción de la acción reflexiva, como se ha visto con el argumento de López-Vargas y Basto-Torrado, 2010, implica considerar una cierta incertidumbre y azar en las actuaciones de los docentes, sin que se caiga en la idea de una no cientificidad sobre el estudio de los modelos pedagógicos y su concreción en prácticas. En esa línea de la incertidumbre, del hacer continuo, se puede considerar la siguiente cita de Mejía: “...la práctica reflexiva genera en los profesores e investigadores el desarrollo de conocimientos no claramente formalizables que ellos luego usarán en sus 
nuevas situaciones pedagógicas, pero siempre desde la actitud de la incertidumbre sobre lo que va a pasar, y la precaución correspondiente" (2005: 75).

Sobre estas consideraciones, se hace necesario pensar en la labor docente como un espacio flexible, que recurre a la experiencia particular, a la reflexión con el fin de lograr los objetivos pedagógicos. Esta flexibilidad debe estar relacionada y puesta en contexto gracias a los procesos reflexivos por parte de los docentes y en la relación complementaria con el mundo de los estudiantes y sus comunidades implícitas. Esto lo afirma Chacón (2006) cuando se hace referencia al poder de la reflexión en el desarrollo de estudiantes comprometidos con sus procesos educativos: "La reflexión como proceso de pensamiento deliberado, racional y crítico se traduce en el examen, la argumentación, la deliberación y toma de decisiones respecto a nuestras actuaciones como docentes" (2006: 338).

En consecuencia, las prácticas pedagógicas basadas en la reflexión y la crítica, según Chacón, se consideran en una oportunidad para el desarrollo de la creatividad, la puesta en común de ideas sobre las teorías y conocimientos, todo ello anclado a una contextualización permanente con las realidades que se viven.

Frente a estas consideraciones sobre la subjetividad, la reflexión, reflejadas en la práctica, Torres de Márquez plantea la posibilidad de la interacción en términos de la construcción colectiva con el fin de construir y transformar las experiencias del estudiante. Según el autor: "El acto pedagógico se constituye en un sistema de interacciones humanas, en el cual estudiantes y profesores ponen de manifiesto conductas que en alguna medida desvelan sus concepciones previas o visiones de mundo, las cuales orientarán de un modo u otro sus acciones en el hecho educativo" (2008: 702).

De este modo, el hecho educativo -el acto- implica el reconocimiento de las historias previas tanto de docentes como de estudiantes, lo cual permite entender que en la participación de los contextos educativos se requiere tanto de la acción del conocimiento, así como la acción de la reflexión y la proposición. Esto tiene correspondencias significativas con las posturas construccionistas sociales, pues se parte de la idea que las historias de las personas están atravesadas por acuerdos narrativos, mediados históricamente, los cuales son permanentemente actualizados en la vida social (Gergen, 1996, 1998, 2006, 2011; Gergen \& Zielke, 2006); en consecuencia, como lo afirma Gergen (frente a la idea general que sustenta su propuesta construccionista): "Quiero proponer un enfoque relacional que considera la autoconcepción no como una estructura cognitiva privada y personal del individuo, sino como un discurso acerca del yo: la representación de los lenguajes disponibles en la esfera pública" (1996: 231).

\section{PRÁCTICAS Y NUEVAS TECNOLOGÍAS: LA CONSTRUCCIÓN SOCIAL DEL SABER EN RED}

Las reflexiones sobre las prácticas pedagógicas en la estrecha relación con la era del conocimiento, se constituye en un campo extenso de posibilidades, unidas muchas veces en la correspondencia con otras disciplinas, sobre todo en el campo de los estudios culturales, especialmente con los aportes de Hall sobre el efecto ideológico de los medios de comunicación y sus prácticas sociales (Hall, 2010); los aportes de Levy sobre cibercultura e inteligencia colectiva $(2007,2004,2002,1998)$ y otra serie de consideraciones teóricas que llaman la atención a esta era como un eje importante para comprender la realidad 
actual. Sin ir más lejos, en el plano de los estudios culturales, Bermúdez y Martínez (2001) plantean la importancia de considerar el estudio del ciberespacio en términos de nuevas sociabilidades, de nuevas experiencias de relación y nuevas consideraciones metodológicas.

En el mismo sentido, Levy, al hablar de cibercultura, plantea la necesidad de comprender las transformaciones de lo virtual, de la ubicuidad del conocimiento, de la necesidad de la cooperación para comprender el paso a la cibercultura. En sus palabras: "Ubicuidad de la información, documentos interactivos interconectados, telecomunicación recíproca y asíncrona de grupo y entre grupos: el carácter virtualizador y desterritorializador del ciberespacio hace de esto el vector de un universal abierto". (2007: 36).

La propuesta hecha desde Levy permite observar que en los espacios de interacción educativa es relevante comprender las transformaciones que se dan en la transmisión del conocimiento, teniendo como referente el ámbito virtual con una serie de connotaciones específicas que interconectan a sus cibernautas en un mundo abierto a muchas posibilidades informativas, que involucran no solo las tecnologías habituales centradas en la escritura tradicional, sino nuevos modos de práctica letrada (integradas en el hipertexto, la hipermedialidad, la escritura en red, entre otros). Al respecto y como ejemplo, de este tipo de nuevas interacciones se puede mencionar los aportes de Casany (2008) sobre las nuevas práctica letradas, en los que se involucran dispositivos digitales de lectura, las correspondencias entre estos dispositivos y las consecuencias en los modos de lectura y escritura de estos nuevos lectoautores (término acuñado por Landow $(1995,1997)$ ) y que resulta muy actual a estas nuevas relaciones entre autor, texto y lector.

Frente a estas consideraciones, las investigaciones que intentan comprender las relaciones de las prácticas pedagógicas en estos escenarios hipertextuales y mediados por las tecnologías recientes, se vuelven cada vez más significativas. Por ejemplo, en el estudio realizado por Valenzuela (2006), se investigó la implementación de las TIC en 22 unidades educativas de la provincia de Nuble, Chile. A través de una metodología descriptiva y de estudio de caso, se identificó que existe una apropiación de las diversas tecnologías, pero estas fueron aplicadas más en el contexto personal y administrativo, dejando el uso de estas tecnologías en las prácticas pedagógicas en un nivel meramente transmisor de información, con una extensión de uso de dos horas a la semana aproximadamente. Dentro de las conclusiones de la investigación se plantea que:

Los modelos que incorporan la dimensión del conocimiento pedagógico presentan de mejor forma los ámbitos en donde se requiere una mayor intervención. Ello significa incorporar en el perfeccionamiento docente instancias de reflexión sobre el potencial que tienen las TIC, no solo para hacer más eficiente el proceso de enseñanza y aprendizaje, sino también para extenderlo y transformarlo (2006: 8).

Esto implica que la relación de las prácticas pedagógicas con los modelos de donde surgen deben estar en sincronía con el uso, implementación y transformaciones de las tecnologías de información y comunicación, pues no es suficiente el conocimiento que los docentes tienen de estas tecnologías; debe reconocerse en el contexto las potencialidades y complejidades de su relación a partir de situaciones cotidianas. Resulta importante reconocer que el uso implica el paso de lo personal a lo educativo, lo que implica ver que de la experiencia concreta de sus usos en los espacios personales se pueden extraer estrategias para que puedan ser volcadas en el contexto educativo y de formación. 
En la misma línea, Barros, Chavarría y Paredes (2008), en la necesidad de identificar las transformaciones de las prácticas pedagógicas gracias al uso de las TIC en los procesos de enseñanza, han diseñado un modelo de análisis como producto de un proceso de investigación de orden cualitativo. En dicho análisis se plantean, en primer lugar, preguntas que haría el docente sobre el cambio de sus creencias para los usos de las TIC y cómo se han integrado en sus prácticas. A partir de las dudas, de los interrogantes, se plantea el proceso de cambio de creencia, lo que implica pensar que si no existen dudas, no hay cambio posible. Este análisis implica la valoración del docente y sus dudas como generadoras de cambio, por lo que es preciso que se conduzca al cambio gracias a la incertidumbre. Ello requiere de la participación reflexiva y de la incorporación de las TIC en el trabajo autónomo del estudiante. En palabras de los autores: "Cuando el profesor valora la indagación personal o cree en la posibilidad de que los estudiantes construyan ciencia, las TIC pueden ayudar a organizar un entorno de aprendizaje más rico" (2008: 68).

\section{DISCUSIÓN: LAS PRÁCTICAS EN EL SENO DE LAS RELACIONES MEDIADAS NARRATIVAMENTE}

Una propuesta renovadora sobre prácticas pedagógicas, es recogida en la reflexión que propone Aiello (2005), en la que se argumenta la necesidad de abordar el análisis y la investigación de las prácticas de la enseñanza como un objeto de estudio, específicamente desde la etnografía, en tanto metodología emergente. Estas metodologías, en general, aluden a la comprensión de los fenómenos humanos a partir de la experiencia narrativa de sus integrantes, de la interpretación de sus puntos de vista, de la reflexión constante sobre sus experiencias consigo mismos y con otros. En este sentido, desde una metodología cualitativa, etnográfica, el énfasis, de acuerdo a la autora, implica considerar que: "Desde este enfoque se entiende que la educación se plantea y se construye desde el aula, sin dejar de tener en cuenta la teoría, en un proceso de reconstrucción permanente, no desde grandes modelos educativos, sino a partir de las prácticas" (2005: 331).

Dentro de estas experiencias de investigación, en la producción de sentidos e interpretaciones sobre el quehacer del docente, se puede incluir entre otros, el estudio de Santamaría et al. (2009) sobre los sentidos que construyen los maestros sobre formación y aprendizaje; se resume en que la experiencia de los docentes implica no pensar en una sola forma de discurso, sino en la diversidad de historias y recursos que se tejen alrededor de la experiencia de clase, lo que hace que el concepto de práctica se fortalezca. En palabras de los autores: “...la práctica pedagógica también se instala sobre un conjunto de significados derivados del contexto social, los cuales regulan los enunciados cotidianos del hablante maestro y estudiante" (2009: 90).

Si se revisan las apreciaciones de Díaz, Martínez, Roa y Sanhueza (2010), sobre la relación del sistema cognitivo desde una perspectiva socio constructivista, resulta valioso considerar el concepto de creencia en las prácticas pedagógicas, relación que tiene notables repercusiones en las experiencias de aula; para los autores, resulta importante tener en cuenta estos sistemas de creencias, dada la innegable correspondencia con las prácticas, la experiencia previas de los docentes, sus historias como estudiantes, su formación profesional, entre otros. Por consiguiente, el siguiente argumento ayuda a comprender el 
sentido de las aproximaciones cualitativas en el contexto educativo, al rescatar sus bondades y mostrar formas alternativas de conocimiento dentro de los espacios educativos:

Investigar los pensamientos y conocimientos de profesores/as significa comprender las concepciones, creencias, dilemas, teorías que gobiernan la práctica profesional e identificar los procesos que constituyen el aprender a enseñar y las categorías conceptuales en las que se articula el conocimiento básico para desarrollar la actividad profesional de la enseñanza (2010: 431).

Resulta claro considerar los aportes de las teorías cognitivas para el fortalecimiento de las prácticas dentro del aula, además del sentido discursivo que permea las prácticas cotidianas sobre el proceso de enseñanza aprendizaje. Esto lo confirma Díaz y Solar (2009) al referirse a la importancia de la actividad cognitiva del docente, en su relación personal, histórica y contextual. Gracias a las construcciones pedagógicas centradas en eventos e historias el docente puede construir un esquema que organiza la información, lo cual le orienta a organizar dicha información en conocimientos prácticos, a reconocer las experiencias particulares de los estudiantes, a ser permeado de las historias previas sobre la enseñanza y satisfacer los retos que los contextos demandan. En palabras de los autores: "Las construcciones pedagógicas se originan producto de un proceso inventivo que es influenciado por la interacción entre conocimientos y creencias de diversas categorías. El conocimiento pedagógico personal es el resultado de la interacción de diferentes categorías de conocimientos y creencias (2009: 51)".

También se puede identificar como relevante el uso de estrategias de capacitación y otras estrategias de carácter constructivista para el mejoramiento y actualización de las prácticas de los docentes. Como ejemplo de esto, se puede mencionar el estudio de Denegri (2005), en el que se realiza una capacitación sobre proyectos de aula en docentes de cuatro establecimientos de educación media en la Región de la Araucanía, Chile. Se demostró frente a los aportes cualitativos, gracias a la utilización de grupos focales y entrevistas a profundidad que:

Tal como se refleja en los resultados de las redes semánticas y en las entrevistas, se observa la reconstrucción de un concepto de aprender y enseñar más cercano a un paradigma constructivista y se manifiesta consistencia entre el discurso y la práctica de los profesores, ya que manejan un bagaje conceptual que se refleja en su quehacer habitual (2005: 45).

Para que se conciba esta legitimación de las puestas en común sobre los discursos involucrados en las prácticas pedagógicas, como se expondrá en el argumento de Patiño-Garzón y Rojas-Betancur (2009), es preciso detenerse un instante en la necesidad de entender el contexto en el que se enuncia la práctica, además del reconocimiento de la experiencia discursiva de sus participantes. Al respecto, Van Dijk (2008), plantea la importancia de integrar el contexto en la perspectiva de construcción interactiva del discurso. Así:

...los contextos, al igual que el discurso, no son objetivos, en el sentido de que están constituidos por hechos sociales que todos los participantes interpretan y consideran relevantes de la misma manera. Son interpretados o construidos, y estratégica y continuamente producidos como hechos relevantes por y para los participantes (2008: 38).

De lo anterior, la variedad y recursividad que se manifiesta en la disposición de los participantes del discurso sobre la novedad de una práctica pedagógica, implica no solo el acuerdo intersubjetivo sobre estas transformaciones en el quehacer del aula, sino que se debe involucrar la historia anterior de otras relaciones, otros contextos y otras percepciones 
sociales sobre el sentido de la práctica. En definitiva, el acuerdo, el consenso, permite que la riqueza de las nuevas prácticas sea un recurso para el aprendizaje comprometido.

En consecuencia, la mirada sobre la práctica pedagógica, de acuerdo a Patiño-Garzón y Rojas-Betancur (2009), se concibe como un proceso en el que actúan diferentes nociones, legitimaciones y puestas en común. Así: "La práctica pedagógica se concibe como un proceso gradual, que se legitima en el campo aplicado y que asume, de esta manera, la validación de la teoría educativa, la construcción de la didáctica y la incorporación de la ciencia y la tecnología al quehacer docente" (2009: 101).

Resulta relevante pensar en la subjetivación de la acción docente como significativa para el desarrollo de interacciones en el aula en su definición más abierta posible. Espacio que convoca la relación cara a cara, la mediación tecnológica y las formas de control, sus tecnologías, que están presentes en cualquier relación interpersonal e institucional. En este orden de ideas, las diferentes estrategias pedagógicas (tanto las tradicionales como las medidas por las actuales tecnologías de la información y la comunicación) establecen un diálogo desde la incertidumbre inicial, en el sentido de un recurso que activa nuevas relaciones y puestas en común sobre los objetivos de aprendizaje. De igual forma, tendrían cabida las experiencias que se puedan construir desde otras disciplinas.

Dentro de las aproximaciones interdisciplinares sobre las prácticas pedagógicas, Romero (2010) plantea relaciones significativas de esas prácticas (desde una perspectiva foucaltiana) en el contexto educativo y, sobre todo, en la potencia de este concepto en la formación de la subjetividad. De acuerdo con la autora:

...la cultura en un contexto local, democrático, en la cotidianidad del aula y de la institución educativa permite reconfigurar prácticas hacia el intercambio y transformación del conocimiento y de su experiencia, en tanto la labor del profesor no pretende desarrollar la motivación hacia el aprendizaje, sino la necesidad de generar tareas de aprendizaje dentro de una cultura que adquiere un significado negociado y compartido (2010: 147).

De lo anterior, estas aportaciones desde Foucault permiten comprender el sentido de la práctica como un saber de saberes que estructura metodológica y epistemológicamente el desarrollo de la educación y las acciones propias dentro de los contextos educativos, al integrar metodologías, conceptos y prácticas derivadas de otros campos de conocimiento. En este sentido, la experiencia interdisciplinar se constituye en la mayor apuesta para la comprensión de las prácticas pedagógicas en la renovación de sus principios operadores, gracias al continuo trabajo de comprensión crítica que ofrecerían sus actores.

En consecuencia, los aportes del estudio del discurso a las prácticas pedagógicas implica reconocer que la acción mediata en la práctica pedagógica requiere de una aproximación cualitativa a la experiencia del aula, en la que se disponen de forma activa diferentes niveles de realidad sobre el objetivo de enseñanza, lo cual implica la postura comprensiva y atenta, tanto de los estudiantes, docentes, y de los desplazamientos institucionales que permitan que estos movimientos se produzcan. Específicamente, la mirada que ofrece el análisis mediato del discurso (AMD) -una de las expresiones metodológicas del análisis del discurso (AD)- permite entender que la acción de la práctica social, en este caso la práctica pedagógica, implica el entrecruzamiento de diferentes prácticas, situadas en un hecho social específico. En palabras del autor: "Una acción mediata no es una clase de acción, sino más bien el momento en tiempo real en el que una serie de múltiples prácticas sociales se entrecruzan para constituir un momento único en la historia, momento que los participantes identifican como una acción social" (2003: 215). 
De este modo, gracias al estudio de cómo se hallan interconectadas las acciones sociales en las actividades cotidianas de las personas, en la generalidad del discurso sobre la vida social, es que este tipo de análisis resulta valioso para estudiar la correspondencia de las actuaciones de la institución educativa, en la experiencia concreta del aula, además de reconocer el sentido y consecuencias de las acciones de sus participantes en el seno de las relaciones sociales.

Otra de las aproximaciones discursivas que resultan significativas para la comprensión de las prácticas pedagógicas tiene que ver con el análisis crítico del discurso (ACD); en esta propuesta metodológica, de acuerdo a Fairclough y Wodak (2008), "El hecho de describir el discurso como práctica social sugiere una relación dialéctica entre un suceso discursivo particular y las situaciones, instituciones y estructuras sociales que lo enmarcan" (367). Mientras que la acción mediata ve la complejidad de la acción cotidiana en sus relaciones intrínsecas, el análisis crítico del discurso ve las relaciones estructurales que sobrepasan a esta acción, en la relación complementaria con las prácticas discursivas generales. Además, esta lectura crítica enfatiza sus lecturas en las relaciones de poder que se instalan en las acciones sociales, lo cual otorga un carácter ideológico al que no puede escaparse el investigador de estos procesos.

\section{CONCLUSIONES: LA PSICOLOGÍA CULTURAL Y SUS PROCESOS DE CONSTRUCCIÓN CRÍTICA DE LA PRÁCTICA PEDAGÓGICA.}

Con el fin de establecer algunas consideraciones finales sobre las prácticas pedagógicas, bajo una mirada construccionista social y enfatizada a una psicología cultural, se reflexiona a continuación sobre los retos y perspectivas de desarrollo conceptual y metodológica que permitan hacer lecturas contextualizadas y críticas de este tema tan actual y sensible en los procesos educativos.

En primer lugar, es necesario considerar las significaciones de los espacios simbólicos, de los contextos históricos sociales, producto de las transformaciones postmodernas, en tanto espacios de análisis para las prácticas pedagógicas. Esta idea, expresada en los argumentos de Cascante y González (2008), puede convocar a la actualización del saber en términos de un espacio revitalizador de la experiencia humana. Así:

[sobre la necesidad de reconocer de la influencia recíproca de historia, sistema educativo y práctica pedagógica como espacio de análisis de todo docente]: Ello implica el ejercicio permanente de la reflexión crítica sobre la realidad, la cual se inicia con la reflexión particular de sus experiencias como docente, ciudadano y persona; transcurre con la valoración de otros juicios colectivos y aterriza con la pertinencia de desnudar las exigencias que se le demandan a la educación, a la luz de la necesidad de conformar sociedades más justas e inclusivas (2008: 62).

En segundo lugar, se reconocen los aportes de Levy sobre el sentido del aprendizaje, en cuanto a la concreción de un espacio educativo que tenga por objeto la consolidación de una inteligencia colectiva que brinde la importancia a la valorización del saber de las personas y la reciprocidad que de este surge en la interacción y responsabilidades especiales, en este caso, atribuidas al docente como motor de la interacción en el proceso de enseñanza aprendizaje. Así: 
El docente se convierte en animador de la inteligencia colectiva de los grupos que tiene a su cargo. Su actividad se centrará en el acompañamiento y la gestión de los aprendizajes: la incitación al intercambio de saberes, la mediación racional y simbólica, el pilotaje personalizado de los recorridos de aprendizaje, etcétera (2007: 144).

Derivado de lo anterior, la actividad mediada por las TIC es importante también en el desarrollo y potenciación de las prácticas pedagógicas. En la investigación de Coll, Onrubia y Mauri (2007) se concluye que: "gracias a la incorporación de las TIC, las formas de organización de la actividad conjunta cambian y se transforman; estas tecnologías hacen efectiva su potencialidad para mediar los procesos psicológicos implicados en la construcción del conocimiento" (399).

En tercer lugar, gracias a los aportes de la psicología cultural de Cole (1999), los procesos de la actividad conjunta, de la relación de artefactos en sus procesos de mediación y las nociones de actividad y práctica, se puede afirmar que constituyen referentes importantes para la lectura complementaria y diversa de las prácticas, no solo en el caso de las TIC, sino, en general, de las relaciones interpersonales construidas en el aula de clase y en los procesos de enseñanza y aprendizaje. Como el autor afirma, sería necesario, para poner la cultura en el centro, considerar que "la mediación cultural implica un modo de cambio evolutivo en el que las actividades de generaciones anteriores se acumulan en el presente como la parte específicamente humana del ambiente" (1999: 137). En consecuencia, desde esta perspectiva, cada cambio en la forma como se construyen las relaciones educativas, expresadas en sus prácticas, ofrece la complejidad de su historia (al reconocerla) y brinda la posibilidad de su transmutación a nuevas correspondencias centradas en el uso de TIC, relaciones discursivas que van más allá del aula de clase y exigen de sus participantes la crítica y auto referencia constante de sus actuaciones, en tanto seres relacionales.

A tono con lo anterior, los ejes para una lectura cultural y crítica de las prácticas pedagógicas, implican, entre otros, el reconocimiento de las acciones particulares de las personas a través de una lectura institucional e histórica de sus repercusiones específicas (Potter, 2008); el realizar críticas sobre considerar a las escuelas como universos cerrados, netamente institucionales (Pizzinato, 2009); construir programas educativos que recrean, a partir de la imaginación y la creatividad, los universos culturales de los jóvenes (Perinat y Tarabay, 2008). Asimismo, el reconocimiento de la subjetividad como eje estructural de las relaciones, todo ello desde una visión histórico-cultural (González-Rey, 2009). De igual forma, reiterar la aproximación hecha por Rebollo (2002), sobre adoptar una perspectiva de investigación que permita los aportes de las tecnologías de la información y la comunicación en la construcción de la identidad cultural. Resulta también importante considerar los aportes de Ratner, sobre la psicología micro cultural centrada en las relaciones interpersonales, bien sean formales o informales (Esteban y Ratner, 2010).

En resumidas cuentas y como lo sostienen los aportes teóricos de Bruner (2002, 2004) acerca de la naturaleza relacional del yo, de su estructuración en guiones mentales que orientan sus puestas en contexto, la práctica pedagógica se constituye en un escenario para crear nuevas relaciones pedagógicas, extendidas a problemáticas sociales específicas. Esto requiere, como se ha visto, de metodología centradas en el discurso y en la posibilidad de crear nuevas interacciones entre autor, texto y lector (extendidas en el uso de las tecnologías de la información y la comunicación), en tanto posibilidad de extender redes de significado en diversidad de contextos (Rodríguez, 2008). 
Estudios Pedagógicos, vol. XL, n. 1, 359-372, 2014

CONSTRUCCIONES CRÍTICAS SOBRE LAS PRÁCTICAS PEDAGÓGICAS: REFLEXIONES A PARTIR DE LA

PSICOLOGÍA CULTURAL Y DISCURSIVA

\section{REFERENCIAS BIBLIOGRÁFICAS}

Aiello, M. (2005). Las prácticas de la enseñanza como objeto de estudio. Una propuesta de abordaje en la formación docente. Educere, vol. 9, n. 30, 329-332.

Barros, B., Chavarría, M., y Paredes, J. (2008). Para analizar la transformación con tic de la enseñanza universitaria. Un estudio exploratorio sobre creencias pedagógicas y prácticas de enseñanza con tic en universidades latinoamericanas. Reifop, vol. 11, n. 1, 59-70.

Bermúdez, E. y Martínez, G.(2001). Los estudios culturales en la era del ciberespacio. Convergencia, vol. $8, n .26,11-31$.

Bruner, J. (2002). Actos de significado: más allá de la revolución cognitiva. Madrid: Alianza Editorial S.A.

Bruner, J. (2004). Realidad mental y mundos posibles. Los actos de la imaginación que dan sentido a la experiencia. Barcelona: Gedisa.

Cascante, L. y González, F. (2008). Repensar la educación y la pedagogía: algunas reflexiones críticas. Revista Electrónica Educare, vol. 12, n. 2, 47-64.

Casany, D. (2008). Prácticas letradas contemporáneas. México: Ríos de tinta.

Cole, M. (1999). Psicología cultural. Madrid: Morata.

Coll, C., Onrubia, J. y Mauri, T. (2007). Tecnología y prácticas pedagógicas: las TIC como instrumentos de mediación de la actividad conjunta de profesores y estudiante. Anuario de Psicología, vol. 38, n. 3, 377-400.

Chacón, M. (2006). La reflexión y la crítica en la formación docente. Educere, vol. 10, n. 33, 335-342.

Denegri, M. (2005). Proyectos de aula interdisciplinarios y reprofesionalización de profesores: un modelo de capacitación. Estudios pedagógicos, vol. 31, n. 1, 33-50.

De Vicenzi, A. (2009). Concepciones de enseñanza y su relación con las prácticas docentes: un estudio con profesores universitarios. Educación y educadores, vol. 12, n. 2, 87-101.

Díaz, C., Martínez, P., Roa, I. y Sanhueza, M. (2010). Los docentes en la sociedad actual: sus creencias y cogniciones pedagógicas respecto al proceso didáctico. Polis, vol. 9, n. 25, 421-436.

Díaz, C. y Solar, M. (2009). Los procesos de cognición como herramienta de la cultura docente. Theoria, vol. 18, n. 2, 43-54.

Esteban, M. y Ratner, C. (2010). Historia, conceptos fundacionales y perspectivas contemporáneas en psicología cultural. Revista de historia de la psicología, vol. 31, n. 2-3, 117-136. Consultado el día 10 de agosto de 2012 de: http://www.sonic.net/ cr2/psicologia\%20cultural10.pdf

Mejía, A. (2005). Hacia una investigación en pedagogía sin tanta ciencia (y con más filosofía). Revista de estudios sociales, $n$. 20, 69-79.

Fairclough, N. y Wodak, R. (2008). Análisis crítico del discurso. En T. Van Dijk (Comp.), El discurso como interacción social. Estudios sobre el discurso II. Una introducción multidisciplinaria. Barcelona: Gedisa.

Fernández Rodríguez, Eduardo (2005). Un Análisis Político de los Discurso Curriculares en la Universidad: Pedagogía crítica sin competencias ... pero con principios de procedimiento. Reifop, 8(2). Consultada en el día 10 de agosto de 2012 de: http://www.aufop.com/aufop/home/

Francis, S. y Marín, P. (2010). Hacia la construcción del saber pedagógico en las comunidades académicas: un estudio desde la opinión de docentes universitarios. Revista Electrónica Actualidades Investigativas en Educación, vol. 10, n. 2, 1-29.

Gergen, K. (1996). Realidades y relaciones. Aproximaciones a la construcción social. Madrid: Paidós.

Gergen, K. (1998). From control to coconstruction: new narratives for the social sciences. Psychological Inquiry, vol. 9, n. 2, 101-103. Actualizado el día 25 de enero de 2009 de: http:// www.jstor.org/stable/1449100.

Gergen, K. (2006). El yo saturado. Dilemas de identidad en el mundo contemporáneo. Madrid: Paidós.

Gergen, K. \& Zielke, B. (2006). Theory in Action. Theory Psychology, vol. 16, n. 3, 299-309. Recuperado el día 24 de octubre de 2009 de: http://tap.sagepub.com/cgi/content/short/16/3/299. 
Gergen, M. y Gergen, K. (2011). Reflexiones sobre la construcción social. Barcelona: Gedisa.

González-Rey, F. (2009). Las categorías de sentido, sentido personal y sentido subjetivo en una perspectiva histórico-cultural: un camino hacia una nueva definición de subjetividad. Universitas Psychologica, vol. 9, n. 1, 241-253. Consultado el día 10 de agosto de 2012 de: http://redalyc. uaemex.mx/redalyc/pdf/647/64712156019.pdf

Hall, S. (2010). Sin garantías. Trayectorias y problemáticas en estudios culturales. En Retrepo, E., C. Walsh y V. Vich (Eds.), Universidad Andina Simón Bolívar, Pontificia Universidad Javeriana, Instituto de Estudios Peruanos. Envión editores: Popayán.

Landow, G. (1995). Hipertexto. La convergencia de la teoría crítica contemporánea y la tecnología. Madrid: Paidós.

Landow, G. (1997). Teoría del hipertexto. Madrid: Paidós.

Latorre, M. (2004). Aportes para el análisis de las racionalidades presentes en las practicas pedagógicas. Estudios pedagógicos, n. 30, 75-91.

Levy, P. (2007). Cibercultura, la cultura de la sociedad digital. México: Anthropos.

Levy, P. (2004). Inteligencia colectiva: por una antropología del ciberespacio. Washington: OPS.

Levy, P. (2002). Ciberdemocracia. Barcelona: Paidós.

Levy, P. (1998). ¿Qué es lo virtual? Barcelona: Paidós.

López-Vargas, B. y Basto-Torrado, S. (2010). Desde las teorías implícitas a la docencia como práctica reflexiva. Educación y Educadores, vol. 13, n. 2, 275-291.

Martínez-Otero, V. (2010). Modelo pentadimensional del discurso educativo aplicado a la educación a distancia. Praxis educacional, vol. 6, n. 8, 107-122. Consultado el día 10 de agosto de 2012 de: http://periodicos.uesb.br/index.php/praxis/article/viewFile/289/322

Patiño-Garzón, L. y Rojas-Betancur, M. (2009). Subjetividad y subjetivación de las prácticas pedagógicas en la universidad. Educación y Educadores, vol. 12, n. 1, 93-105.

Perinat, A. y Tarabay, F. (2008). Educación y desarrollo humano en América Latina; reflexiones desde la psicología cultural. Universitas Psychologica, vol. 7, n. 3, 701-710. Consultado el día 10 de agosto de 2012 de: http://sparta.javeriana.edu.co/psicologia/publicaciones/actualizarrevista/archivos/V07N03A07.pdf

Pizzinato, A. (2009). Psicología cultural. Contribuciones teóricas y fundamentos epistemológicos de las aportaciones de Vygotsky hacia la discusión lingüística de Bakhtin. Universitas Psychologica, vol. 9, n. 1, 255-261. Consultado el día 10 de agosto de 2012 de: http://redalyc. uaemex.mx/pdf/647/64712156020.pdf

Potter, J. (2008). Hacer que la psicología sea relevante. Discurso y sociedad, vol. 2, n. 1, 186200. Consultado el día 10 de agosto de 2012 de: http://www.dissoc.org/ediciones/v02n01/ DS2\%281\%29Potter.pdf

Prados, M. y Cubero, R. (2005). Construcción del conocimiento y discurso educativo. Una aproximación al estudio del discurso de profesores y alumnos en la universidad. Avances en psicología latinoamericana, n. 23, 141-153. Consultado el día 10 de agosto de 2012 de: http://redalyc. uaemex.mx/pdf/799/79902311.pdf

Quiroz, R. (2006). La enseñanza de las corrientes pedagógicas: una propuesta didáctica desarrolladora, Íkala, revista de lenguaje y cultura, vol. 11, n. 17, 339-361.

Rebollo, M. (2002). La investigación educativa sobre nuevas tecnologías: una aproximación sociocultural. Enseñanza, n. 20, 113-126. Consultado el día 10 de agosto de 2012 de: http://e-spacio. uned.es/fez/eserv.php?pid=bibliuned:20372\&dsID=investigacion_educativa.pdf

Rodríguez, A. (2008). El análisis del discurso y sus aportaciones a los estudios literarios en el marco de las coordenadas autor, obra, lector y contexto. Andamios, vol. 5, n. 9, 77-98. Actualizado el día 16 de enero de 2010 de: http://www.scielo.org.mx/pdf/anda/v5n9/v5n9a4.pdf.

Romero, O. (2010). Constitución de sujeto-profesor universitario a través de las prácticas de enseñanza. Revista Virtual Universidad Católica del Norte, n. 31, 131-154. 
Torres de Márquez, A. (2008). La reflexión, la contestación, la proposición y la acción como espacios indispensables en el contexto áulico. Educere, vol. 12, n. 43, 697-705.

Tuñón, M. y Pérez, M. (2009). Características del discurso en el aula de clase como mediación para el desarrollo de pensamiento crítico. Zona próxima, vol. 11, 44-159.

Santamaría, L., Aristizábal, O., Gómez, R., Escobar, R., López, M. Castelblanco, J. Cifuentes, C., Ospina, L. y Murillo, J. (2009). Sentidos que subyacen a las prácticas pedagógicas de maestros de la escuela normal. Revista Latinoamericana de Estudios Educativos, vol. 5, n. 1, 71-95.

Scollon, R. (2003). Acción y texto: para una comprensión conjunta del lugar del texto en la (inter) acción social, el análisis mediato del discurso y el problema de la acción social. En R. Wodak \& M. Meyer, (Eds.), Métodos de análisis crítico del discurso. Barcelona: Gedisa.

Valenzuela, N. (2006). Cómo los modelos de cambio e innovación curricular pueden ayudarnos a comprender el fenómeno de la implementación e integración de las TIC en las prácticas docentes. Resultados de una investigación en 22 unidades educativas de la provincia de Nuble. Horizontes educacionales, $n$. 11, 1-11.

Van Dijk, T. (2011). Sociedad y discurso. Cómo influyen los contextos sociales sobre el texto y la conversación. Barcelona: Gedisa.

Van Dijk, T. (2008). El discurso como interacción en la sociedad. En T. Van Dijk (Comp.), El discurso como interacción social. Estudios sobre el discurso II. Una introducción multidisciplinaria. Barcelona: Gedisa.

Van Dijk, T. (2007). Estructuras y funciones del discurso. México: Siglo XXI. 